\title{
Giant Negative Magnetoresistance Driven by Spin-Orbit Coupling at the $\mathrm{LaAlO}_{3} / \mathrm{SrTiO}_{3}$ Interface
}

\author{
M. Diez, ${ }^{1}$ A. M. R. V. L. Monteiro, ${ }^{2}$ G. Mattoni, ${ }^{2}$ E. Cobanera, ${ }^{1,3}$ T. Hyart, ${ }^{1,4}$ E. Mulazimoglu, ${ }^{2}$ N. Bovenzi, ${ }^{1}$ \\ C. W. J. Beenakker, ${ }^{1}$ and A. D. Caviglia ${ }^{2}$ \\ ${ }^{1}$ Instituut-Lorentz, Universiteit Leiden, P.O. Box 9506, 2300 RA Leiden, Netherlands \\ ${ }^{2}$ Kavli Institute of Nanoscience, Delft University of Technology, Lorentzweg 1, 2628 CJ Delft, Netherlands \\ ${ }^{3}$ Institute for Theoretical Physics, Center for Extreme Matter and Emergent Phenomena, Utrecht University, \\ Leuvenlaan 4, 3584 CE Utrecht, Netherlands \\ ${ }^{4}$ Department of Physics and Nanoscience Center, University of Jyväskylä, P.O. Box 35 (YFL), \\ FI-40014 University of Jyväskylä, Finland
}

(Received 16 January 2015; published 2 July 2015)

\begin{abstract}
The $\mathrm{LaAlO}_{3} / \mathrm{SrTiO}_{3}$ interface hosts a two-dimensional electron system that is unusually sensitive to the application of an in-plane magnetic field. Low-temperature experiments have revealed a giant negative magnetoresistance (dropping by 70\%), attributed to a magnetic-field induced transition between interacting phases of conduction electrons with Kondo-screened magnetic impurities. Here we report on experiments over a broad temperature range, showing the persistence of the magnetoresistance up to the $20 \mathrm{~K}$ rangeindicative of a single-particle mechanism. Motivated by a striking correspondence between the temperature and carrier density dependence of our magnetoresistance measurements we propose an alternative explanation. Working in the framework of semiclassical Boltzmann transport theory we demonstrate that the combination of spin-orbit coupling and scattering from finite-range impurities can explain the observed magnitude of the negative magnetoresistance, as well as the temperature and electron density dependence.
\end{abstract}

DOI: 10.1103/PhysRevLett.115.016803

PACS numbers: 73.40.-c, 74.25.F-, 75.47.-m, 75.70.Tj

The mobile electrons at the $\mathrm{LaAlO}_{3} / \mathrm{SrTiO}_{3}(\mathrm{LAO} / \mathrm{STO})$ interface [1] display an exotic combination of superconductivity [2,3] and magnetic order [4-7]. The onset of superconductivity at sub-Kelvin temperatures appears in an interval of electron densities where the effect of Rashba spin-orbit coupling on the band structure at the Fermi level is strongest $[8,9]$, but whether this correlation implies causation remains unclear.

Transport experiments above the superconducting transition temperature have revealed a very large ("giant") drop in the sheet resistance of the LAO/STO interface upon application of a parallel magnetic field [10-13]. An explanation has been proposed $[13,14]$ in terms of the Kondo effect: Variation of the electron density or magnetic field drives a quantum phase transition between a highresistance correlated electronic phase with screened magnetic impurities and a low-resistance phase of polarized impurity moments. The relevance of spin-orbit coupling for magnetotransport is widely appreciated [10,14-19], but it was generally believed to be too weak an effect to provide a single-particle explanation of the giant magnetoresistance.

In this work we provide experimental data (combining magnetic field, gate voltage, and temperature profiles for the resistance of the LAO/STO interface) and theoretical calculations that support an explanation fully within the single-particle context of Boltzmann transport. The key ingredients are the combination of spin-orbit coupling, band anisotropy, and finite-range electrostatic impurity scattering.
The thermal insensitivity of the giant magnetoresistance $[10,11]$, in combination with a striking correspondence that we have observed between the gate voltage and temperature dependence of the effect, are features that are difficult to reconcile with the thermally fragile Kondo interpretationbut fit naturally in the semiclassical Boltzmann description.

We first present the experimental data and then turn to the theoretical description. Devices were fabricated by using amorphous LAO (a-LAO) as a hard mask and epitaxially depositing a thin (12 u.c.) film of $\mathrm{LAO}$ on top of a $\mathrm{TiO}_{2}$ terminated (001)STO single crystal substrate. The film was grown by pulsed laser deposition at $770{ }^{\circ} \mathrm{C}$ in $\mathrm{O}_{2}$ at a pressure of $6 \times 10^{-5} \mathrm{mbar}$. The laser fluence was $1 \mathrm{~J} \mathrm{~cm}^{-2}$ and the repetition rate was $1 \mathrm{~Hz}$. The growth of the film was monitored in situ using reflection high energy electron diffraction (RHEED), and layer-by-layer growth was confirmed. After deposition, the sample was annealed for $1 \mathrm{~h}$ at $600{ }^{\circ} \mathrm{C}$ in 300 mbar of $\mathrm{O}_{2}$. Finally, the sample was cooled down to room temperature in the same atmosphere. Magnetotransport measurements were performed in a four-probe Hall bar geometry and in a field-effect configuration [Fig. 1(a), inset] established with a homogeneous metallic back gate. The magnetic field $B$ is applied in-plane and perpendicular to the current. The longitudinal sheet resistance $\rho_{x x}(B)$ determines the dimensionless magnetoresistance

$$
\operatorname{MR}(B)=\rho_{x x}(B) / \rho_{x x}(0)-1 .
$$



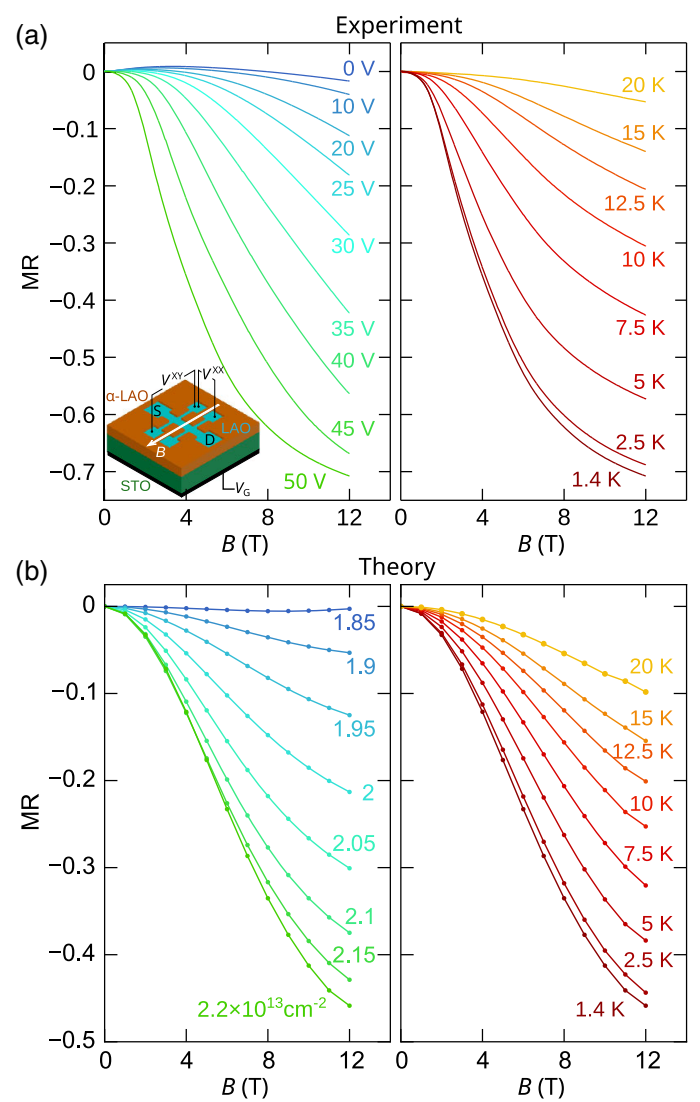

FIG. 1 (color online). (a) Measured magnetoresistance at $T=$ $1.4 \mathrm{~K}$ for different gate voltages (left panel) and at $V_{G}=50 \mathrm{~V}$ for various temperatures (right panel). Inset: Schematic drawing of the device in a Hall bar geometry (in-plane field perpendicular to current direction), showing the source $S$, drain $D$, longitudinal voltage $V^{x x}$, transverse voltage $V^{x y}$ and gate voltage $V_{G}$. (b) Magnetoresistance calculated from the Boltzmann equation, at fixed $T=1.4 \mathrm{~K}$ (left panel) and at fixed $n=2.2 \times 10^{13} \mathrm{~cm}^{-2}$ (right panel).

The left panel of Fig. 1(a) shows the measured magnetoresistance as a function of magnetic field, recorded at $1.4 \mathrm{~K}$, for gate voltages $V_{G}$ ranging from 0 to $50 \mathrm{~V}$. In general, we observe the magnetoresistance to remain mainly flat up to some characteristic value of the magnetic field. For larger values, the magnetoresistance drops sharply. At even higher magnetic fields, the magnetoresistance begins to saturate, producing an overall bell-like curve. At the highest voltage $V_{G}=50 \mathrm{~V}$, a very large negative magnetoresistance is observed (a drop of $70 \%$ ) over a magnetic field range from 0 to $12 \mathrm{~T}$. As $V_{G}$ is decreased, the overall magnitude of the magnetoresistance drop is suppressed, as the curves flatten out and the characteristic field progressively moves to higher $B$. At $V_{G}=10 \mathrm{~V}$, the maximum magnetoresistance variation is less than $5 \%$.

The right panel of Fig. 1(a) shows the measured magnetoresistance at a fixed gate voltage of $V_{G}=50 \mathrm{~V}$, for different temperatures ranging from 1.4 to $20 \mathrm{~K}$. The correspondence between the bell-shaped magnetoresistance profiles as a function of temperature and gate voltage is striking. As $T$ increases or $V_{G}$ decreases, both the magnitude of the magnetoresistance and steepness of $\partial \mathrm{MR} / \partial \mathrm{B}$ decrease. Although the negative magnetoresistance is progressively suppressed as the temperature is raised, it is still clearly visible at $20 \mathrm{~K}$, in agreement with previous experiments $[10,11]$. Notice that the characteristic field scale of the resistance drop increases with temperature.

For the theoretical description we use a three-band model of the $t_{2 \mathrm{~g}}$ conduction electrons at the LAO/STO interface [12], with Hamiltonian

$$
H=\sum_{k, l, l^{\prime}, \sigma, \sigma^{\prime}} c_{k, l, \sigma}^{\dagger}\left(H_{\mathrm{L}}+H_{\mathrm{SO}}+H_{\mathrm{Z}}+H_{\mathrm{B}}\right) c_{k, l^{\prime}, \sigma^{\prime}} .
$$

The operators $c_{k, l, \sigma}^{\dagger}$ create electrons of spin $\sigma$ and momentum $\boldsymbol{k}$ (measured in units of the lattice constant $a=0.4 \mathrm{~nm}$ ), in orbitals $l=d_{x y}, d_{x z}, d_{y z}$ of the Ti atoms close to the interface. We describe the various terms in this three-band Hamiltonian, with parameter values from the literature $[12,20-29]$ that we will use in our calculations. (Further details are given in the Supplemental Material [28].)

The lobes of the $d_{x y}$ orbital are in plane, producing two equivalent hopping integrals $t_{l}=340 \mathrm{meV}$. For the two other orbitals, the $x$ lobe or $y$ lobe is in plane and the $z$ lobe is out of plane, giving rise to one large and one small hopping element $t_{l}=340 \mathrm{meV}$ and $t_{h}=12.5 \mathrm{meV}$, respectively. The $d_{x z}$ and $d_{y z}$ orbitals are hybridized by a diagonal hopping $t_{d}=t_{h}$. Confinement lowers the $d_{x y}$ orbital in energy by $\Delta_{E}=60 \mathrm{meV}$. All this information is encoded in

$$
\begin{aligned}
& H_{L}=\left(\begin{array}{ccc}
\epsilon_{x y}(k)-\Delta_{\mathrm{E}} & 0 & 0 \\
0 & \epsilon_{x z}(k) & \delta(k) \\
0 & \delta(k) & \epsilon_{y z}(k)
\end{array}\right) \otimes \hat{\sigma}_{0}, \\
& \epsilon_{x y}(k)=2 t_{l}\left(2-\cos k_{x}-\cos k_{y}\right), \\
& \epsilon_{x z}(k)=2 t_{l}\left(1-\cos k_{x}\right)+2 t_{h}\left(1-\cos k_{y}\right), \\
& \epsilon_{y z}(k)=2 t_{h}\left(1-\cos k_{x}\right)+2 t_{l}\left(1-\cos k_{y}\right), \\
& \delta(k)=2 t_{d} \sin k_{x} \sin k_{y} .
\end{aligned}
$$

We use $\hat{\sigma}_{x, y, z}$ and $\hat{\sigma}_{0}$ to denote the Pauli matrices and the identity acting on the electron spin.

The intrinsic electric field at the interface breaks inversion symmetry and produces the term

$$
H_{Z}=\Delta_{Z}\left(\begin{array}{ccc}
0 & i \sin k_{y} & i \sin k_{x} \\
-i \sin k_{y} & 0 & 0 \\
-i \sin k_{x} & 0 & 0
\end{array}\right) \otimes \hat{\sigma}_{0}
$$

with $\Delta_{Z}=15 \mathrm{meV}$. Atomic spin-orbit coupling gives 


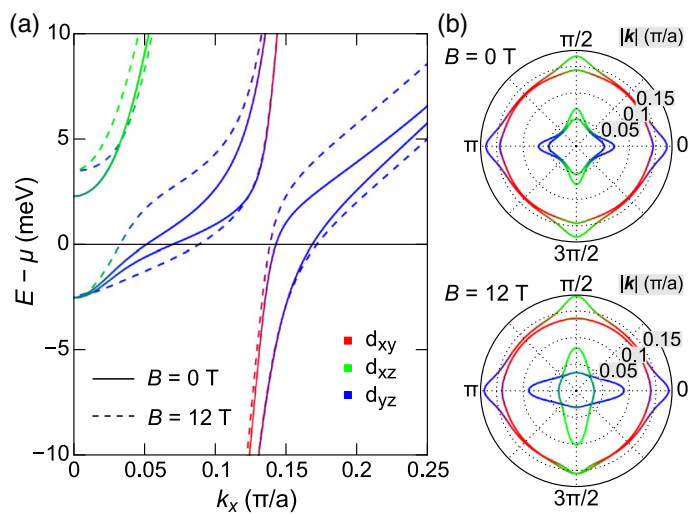

FIG. 2 (color online). (a) Dispersion relation for the mobile electrons at the $\mathrm{LaAlO}_{3} / \mathrm{SrTiO}_{3}$ interface, calculated from the model Hamiltonian (2) for $n=2.2 \times 10^{13} \mathrm{~cm}^{-2}$ at $B=0$ (solid line) and $B=12 \mathrm{~T}$ (dashed line). Colors indicate the orbital character of the bands. (b) Corresponding Fermi surfaces when the chemical potential is located at the "sweet spot" above the Lifshitz point where the system becomes very sensitive to changes in carrier density and magnetic field.

$$
H_{\mathrm{SO}}=\frac{\Delta_{\mathrm{SO}}}{2}\left(\begin{array}{ccc}
0 & i \hat{\sigma}_{x} & -i \hat{\sigma}_{y} \\
-i \hat{\sigma}_{x} & 0 & i \hat{\sigma}_{z} \\
i \hat{\sigma}_{y} & -i \hat{\sigma}_{z} & 0
\end{array}\right),
$$

with an amplitude $\Delta_{\mathrm{SO}}=5 \mathrm{meV}$. Together, $H_{Z}$ and $H_{\mathrm{SO}}$ cause a Rashba-type splitting of the bands, coupling the $d_{x y}$ orbital with the $d_{x z / y z}$ orbitals above the Lifshitz point at the bottom of the $d_{x z / y z}$ bands.

The term $H_{B}=\mu_{B}(\boldsymbol{L}+g \boldsymbol{S}) \cdot \boldsymbol{B} / \hbar$, with $g=5$ [27], describes the coupling of the applied magnetic field to the spin and orbital angular momentum of the electrons, where $\boldsymbol{S}=\hbar \hat{\boldsymbol{\sigma}} / 2$ and

$$
\begin{aligned}
L_{x} & =\hbar\left(\begin{array}{ccc}
0 & i & 0 \\
-i & 0 & 0 \\
0 & 0 & 0
\end{array}\right), \quad L_{y}=\hbar\left(\begin{array}{ccc}
0 & 0 & -i \\
0 & 0 & 0 \\
i & 0 & 0
\end{array}\right), \\
L_{z} & =\hbar\left(\begin{array}{ccc}
0 & 0 & 0 \\
0 & 0 & i \\
0 & -i & 0
\end{array}\right) .
\end{aligned}
$$

The resulting highly anisotropic band structure is shown in Fig. 2. Notice the unusually close relevant energy scales: When measured from the bottom of the upper, anisotropic bands, the Fermi energy, spin-orbit coupling induced spinsplitting, Zeeman energy (10 T), and temperature (10 K) all are on the order of $1 \mathrm{meV}$.

We calculate the magnetoresistance from the model Hamiltonian (2) using the semiclassical Boltzmann transport equation for the momentum $\boldsymbol{k}$ and band index $\nu$ dependent distribution function $f_{\boldsymbol{k}, \nu}=f_{0}\left(\epsilon_{\boldsymbol{k}, L}\right)+g_{\boldsymbol{k}, \nu}$. We linearize around the equilibrium Fermi-Dirac distribution $f_{0}$, at temperature $T$ and chemical potential $\mu$ (determined self-consistently to obtain a prescribed carrier density $n$ ). In this way we find the conductivity tensor

$$
\sigma_{i j}=e \sum_{\boldsymbol{k}, \nu}\left(\boldsymbol{v}_{\boldsymbol{k}, \nu}\right)_{i} \partial g_{\boldsymbol{k}, \nu} / \partial E_{j}
$$

in linear response to the electric field $\boldsymbol{E}$. The longitudinal resistivity $\rho_{x x}$ then follows upon inversion of the $\boldsymbol{\sigma}$ tensor. The band structure determines the velocity $v_{k, \nu}=\hbar^{-1} \nabla_{k} \epsilon_{k, \nu}$, which is not parallel to the momentum $\hbar \boldsymbol{k}$ because of the anisotropic Fermi surface.

Calculations of this type are routinely simplified using Ziman's relaxation-time approximation [30,31], but the combination of finite-range scattering and anisotropic band structure renders this approximation unreliable [32]. We have therefore resorted to a numerical solution of the full partial differential equation

$$
\begin{gathered}
-e\left(\boldsymbol{v}_{\boldsymbol{k}, \nu} \cdot \boldsymbol{E}\right) \partial f_{0} / \partial \epsilon_{\boldsymbol{k}, \nu}=(e / \hbar)\left(\boldsymbol{v}_{\boldsymbol{k}, \nu} \times \boldsymbol{B}\right) \cdot \nabla_{\boldsymbol{k}} g_{\boldsymbol{k}, \nu} \\
+\sum_{\boldsymbol{k}^{\prime}, \nu^{\prime}}\left(g_{\boldsymbol{k}, \nu}-g_{\boldsymbol{k}^{\prime}, \nu^{\prime}}\right) q_{\boldsymbol{k} \nu, \boldsymbol{k}^{\prime} \nu^{\prime}} \delta\left(\epsilon_{\boldsymbol{k}, \nu}-\epsilon_{\boldsymbol{k}^{\prime}, \nu^{\prime}}\right) .
\end{gathered}
$$

Elastic impurity scattering enters with a rate

$q_{\boldsymbol{k} \nu, \boldsymbol{k}^{\prime} \nu^{\prime}}=\frac{2}{3} \pi^{3} \hbar^{-1} \delta^{2} \xi^{4} n_{\mathrm{imp}} e^{-\xi^{2}\left|\boldsymbol{k}-\boldsymbol{k}^{\prime}\right|^{2} / 2}\left|\left\langle u_{\boldsymbol{k} \nu} \mid u_{\boldsymbol{k}^{\prime} \nu^{\prime}}\right\rangle\right|^{2}$.

The impurity density $n_{\text {imp }}$ and scattering amplitude $\delta$ drop out of the magnetoresistance (1), so they need not be specified. The scattering potential has correlation length $\xi$, for which we take $2 \mathrm{~nm} \simeq 5$ lattice constants, consistent with experiments on scattering by dislocations [33]. (We will discuss the role of this finite correlation length later on.) Both intraband and interband scattering are included via the structure factor $\left|\left\langle u_{k \nu} \mid u_{k^{\prime} \nu^{\prime}}\right\rangle\right|^{2}$, which takes into account the finite overlap $\left\langle\psi_{\nu}(\boldsymbol{k})|V(\boldsymbol{r})| \psi_{\nu^{\prime}}\left(\boldsymbol{k}^{\prime}\right)\right\rangle$ of the Bloch states $\psi_{\nu}(\boldsymbol{k})=u_{k \nu}(\boldsymbol{r}) e^{i \boldsymbol{k} \cdot \boldsymbol{r}}$ and $\psi_{\nu^{\prime}}\left(\boldsymbol{k}^{\prime}\right)=u_{\boldsymbol{k}^{\prime} \nu^{\prime}}(\boldsymbol{r}) e^{i \boldsymbol{k}^{\prime} \cdot \boldsymbol{r}}$ [34].

The in-plane magnetoresistance resulting from the Boltzmann equation is shown in Fig. 1(b). The similarity in the bell-shaped magnetoresistance curves, with a corresponding dependence on carrier density and temperature, is clear and remarkable in view of the simplicity of the theoretical model. We conclude that a semiclassical singleparticle description can produce a giant magnetoresistance, up to $50 \%$ for a quite conservative choice of parameter values.

Two main ingredients explain how such a large negative magnetoresistance could follow from a model without electron-electron interactions. The first ingredient is the orbital-mixing character of the atomic and inversionsymmetry-breaking spin-orbit coupling terms $H_{\text {SO }}$ and $H_{Z}$. As a result, the spin-orbit splitting is very nonlinear and produces a sweet spot, that is, a narrow range of Fermi energies (carrier densities $n^{*} \simeq 2.2 \times 10^{13} / \mathrm{cm}^{2}$ ) in which the system becomes sensitive to small changes in the density. If the density (or the corresponding gate voltage) is near the sweet spot, the spin-orbit induced band mixing gives rise to a substantial contribution to the (zero-field) resistance stemming from interband scattering. The Zeeman energy in turn favors an alignment of the spin with the magnetic field and drives a highly anisotropic deformation of the Fermi surface 

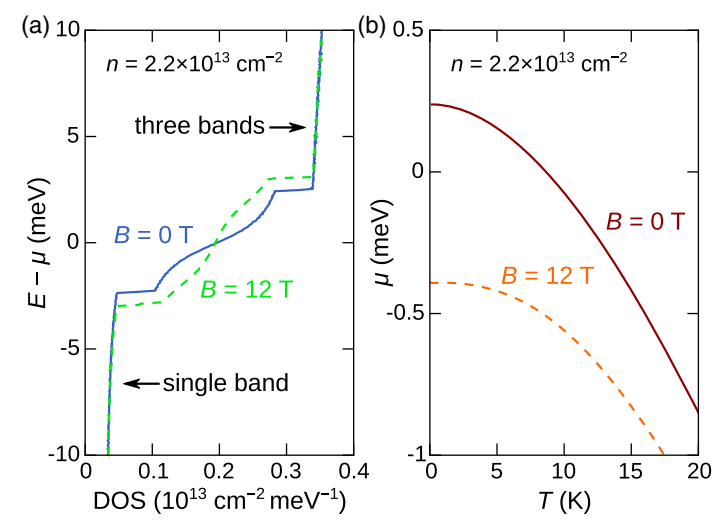

FIG. 3 (color online). Energy-dependent density of states (a) and temperature-dependent chemical potential (b), calculated from the three-band Hamiltonian (2). Both quantities are shown for the sweet-spot carrier density $n=2.2 \times 10^{13} \mathrm{~cm}^{-2}$, at $B=0$ (solid line) and $B=12 \mathrm{~T}$ (dashed line).

into spin-polarized bands (see Fig. 2). Interband scattering is suppressed which explains the decrease in sheet resistance. At densities $n<n^{*}$ only a single band is occupied and spinorbit coupling is well described by a conventional Rashba term $\alpha_{\text {SO }}(\hat{\boldsymbol{\sigma}} \times \boldsymbol{p})[9,22,37]$ and our calculation gives a vanishingly small magnetoresistance. At densities $n>n^{*}$ the calculated magnetoresistance starts to saturate and eventually becomes small again.

The second ingredient is the finite correlation length $\xi$ of the disorder potential. The resulting anisotropic scattering rate (10) is largest at small momenta $\left|\boldsymbol{k}-\boldsymbol{k}^{\prime}\right|$. Moderate values of $\xi$ on the order of a few lattice constants suppress backscattering processes within the outer Fermi surface with large average momentum $k_{F}$, while still allowing for interband scattering. This is accompanied by a quasiparticle lifetime which can be significantly smaller for the inner band (smaller average $k_{F}$ ). The imbalance of band mobilities promotes the importance of interband scattering when transport is dominated by quasiparticles in the outer bands which have a larger Fermi velocity and a small intraband backscattering rate. In comparison, we have found [28] that the isotropic scattering by a delta-function impurity potential cannot produce a magnetoresistance exceeding $15 \%$.

Our theoretical curves show a smooth dependence on temperature, with the negative magnetoresistance persisting beyond $20 \mathrm{~K}$, and they show a striking correspondence between the temperature dependence of the magnetoresistance for a fixed density and the density dependence for a fixed temperature. This correspondence, a hallmark of our experimental data, can be understood as a consequence of the renormalization of the chemical potential as a function of temperature; see Fig. 3. The weak temperature dependence of the Hall resistance points towards a constant carrier density in the range 1-20 K [38]. As shown in Fig. 3(a) the density of states increases steeply with band energy in the vicinity of the sweet spot, much more than in conventional semiconductors. To keep the total carrier density fixed with

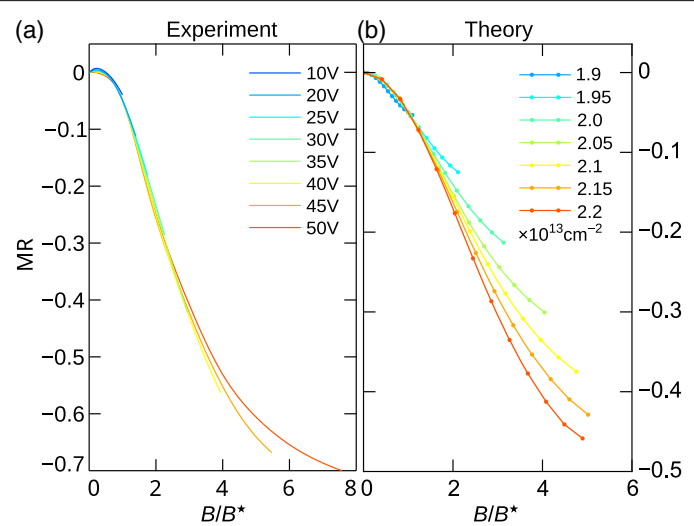

FIG. 4 (color online). Measured (a) and calculated (b) magnetoresistance at $1.4 \mathrm{~K}$ for different densities or gate voltages as a function of the rescaled magnetic field $B / B^{\star}$. The characteristic field $B^{\star}$ is chosen such that the rescaled curves all pass through the point with $\mathrm{MR}=-0.05$.

increasing temperature, the chemical potential is lowered by more than $1 \mathrm{meV}$ at $20 \mathrm{~K}$ compared to its low temperature limit. This is why increasing the temperature is equivalent to probing the band structure at a lower energy, explaining the similarity in the magnetoresistance curves in the left and right panels of Fig. 1.

These are the two key arguments in favor of a singleparticle spin-orbit-coupling based mechanism for the giant negative magnetoresistance: First, the persistence of the effect to elevated temperatures, and second the corresponding effect of temperature increase and density decrease. It seems difficult to incorporate these features of the data in the correlated-electron mechanism [13,14], based on Kondo screening of magnetic moments. There is a third noteworthy feature of the data that is not well reproduced by our calculation, and has been interpreted as evidence for a transition into a low-field Kondo phase [13,14]: A rescaling of the magnetic field $B \rightarrow B / B^{\star}$ by a densitydependent value $B^{\star}$ collapses the measured magnetoresistance at different densities onto a single curve; see Fig. 4(a). If we apply this $B / B^{\star}$ scaling to our numerical results a significant $n$ dependence remains; see Fig. 4(b). The experimental scaling law points to some relevant physics that is not yet included in our minimal model.

In conclusion, we have presented experimental data and theoretical calculations that support a semiclassical singleparticle mechanism for the giant magnetoresistance of the LAO/STO interface. The Boltzman transport equation with spin-orbit coupling, in combination with anisotropy of Fermi surface and scattering rates, suffices to produce a large resistance drop upon application of a magnetic field. The characteristic temperature and carrier-density dependence agrees quite well with what is observed experimentally, but the $B / B^{\star}$ scaling will likely require an extension of the simplest three-band model.

Our explanation of the sudden onset of the magnetoresistance when the carrier density approaches a sweet spot 
of amplified spin-orbit coupling has addressed the normalstate transport above the superconducting transition temperature. Since superconductivity happens in the vicinity of the same sweet spot, it would be interesting to investigate whether spin-orbit coupling plays a dominant role in that transition as well.

We thank A. R. Akhmerov, C. Morais Smith, D. I. Pikulin, J. Ruhmann, and M. Wimmer for fruitful discussions, S. Goswami for support in sample fabrication, and R. Hoogerheide and M. van Oossanen for technical support. This work is part of the DITP and Nanofront consortia, funded by the Netherlands Science Foundation NWO/OCW. We acknowledge support by the Academy of Finland Center of Excellence program, the European Research Council (Grant No. 240362-Heattronics and a Synergy grant), and the Foundation for Fundamental Research on Matter (FOM).

M. D. and A. M. R. V. L. M. contributed equally to this work.

[1] A. Ohtomo and H.-Y. Hwang, Nature (London) 427, 423 (2004).

[2] N. Reyren, S. Thiel, A. D. Caviglia, L. F. Kourkoutis, G. Hammerl, C. Richter, C. W. Schneider, T. Kopp, A.-S. Rüetschi, D. Jaccard, M. Gabay, D. A. Muller, and J.-M. Triscone, Science 317, 1196 (2007).

[3] A. D. Caviglia, S. Gariglio, N. Reyren, D. Jaccard, T. Schneider, M. Gabay, S. Thiel, G. Hammerl, J. Mannhart, and J.-M. Triscone, Nature (London) 456, 624 (2008).

[4] A. Brinkman, M. Huijben, M. van Zalk, J. Huijben, U. Zeitler, J. C. Maan, W. G. van der Wiel, G. Rijnders, D. H. Blank, and H. Hilgenkamp, Nat. Mater. 6, 493 (2007).

[5] J. A. Bert, B. Kalisky, C. Bell, M. Kim, Y. Hikita, H.-Y. Hwang, and K. A. Moler, Nat. Phys. 7, 767 (2011).

[6] L. Li, C. Richter, J. Mannhart, and R. C. Ashoori, Nat. Phys. 7, 762 (2011).

[7] B. Kalisky, J. A. Bert, B. B. Klopfer, C. Bell, H. K. Sato, M. Hosoda, Y. Hikita, H.-Y. Hwang, and K. A. Moler, Nat. Commun. 3, 922 (2012).

[8] M. Ben Shalom, M. Sachs, D. Rakhmilevitch, A. Palevski, and Y. Dagan, Phys. Rev. Lett. 104, 126802 (2010).

[9] A. D. Caviglia, M. Gabay, S. Gariglio, N. Reyren, C. Cancellieri, and J.-M. Triscone, Phys. Rev. Lett., 104, 126803 (2010).

[10] M. Ben Shalom, C. W. Tai, Y. Lereah, M. Sachs, E. Levy, D. Rakhmilevitch, A. Palevski, and Y. Dagan, Phys. Rev. B 80, 140403R (2009).

[11] X. Wang, W. M. Lü, A. Annadi, Z. Q. Liu, K. Gopinadhan, S. Dhar, T. Venkatesan, and Ariando, Phys. Rev. B 84, 075312 (2011).

[12] A. Joshua, S. Pecker, J. Ruhman, E. Altman, and S. Ilani, Nat. Commun. 3, 1129 (2012).

[13] A. Joshua, J. Ruhman, S. Pecker, E. Altman, and S. Ilani, Proc. Natl. Acad. Sci. U.S.A. 110, 9633 (2013).

[14] J. Ruhman, A. Joshua, S. Ilani, and E. Altman, Phys. Rev. B 90, 125123 (2014).

[15] M. Trushin, K. Výborný, P. Moraczewski, A. A. Kovalev, J. Schliemann, and T. Jungwirth, Phys. Rev. B 80, 134405 (2009).
[16] E. Flekser, M. Ben Shalom, M. Kim, C. Bell, Y. Hikita, H. Y. Hwang, and Y. Dagan, Phys. Rev. B 86, 121104R (2012).

[17] A. Fête, S. Gariglio, A. D. Caviglia, J.-M. Triscone, and M. Gabay, Phys. Rev. B 86, 201105R (2012).

[18] S. Caprara, F. Peronaci, and M. Grilli, Phys. Rev. Lett. 109, 196401 (2012)

[19] D. Bucheli, M. Grilli, F. Peronaci, G. Seibold, and S. Caprara, Phys. Rev. B 89, 195448 (2014).

[20] L. F. Mattheis, Phys. Rev. B 6, 4718 (1972).

[21] A. F. Santander-Syro, O. Copie, T. Kondo, F. Fortuna, S. Pailheś, R. Weht, X. G. Qiu, F. Bertran, A. Nicolaou, A. Taleb-Ibrahimi, P. Le Fèvre, G. Herranz, M. Bibes, N. Reyren, Y. Apertet, P. Lecoeur, A. Barthélémy, and M. J. Rozenberg, Nature (London) 469, 189 (2011).

[22] Z. Zhong, A. Tóth, and K. Held, Phys. Rev. B 87, 161102 (2013).

[23] L. W. van Heeringen, G. A. de Wijs, A. McCollam, J. C. Maan, and A. Fasolino, Phys. Rev. B 88, 205140 (2013).

[24] C. Cancellieri, M. L. Reinle-Schmitt, M. Kobayashi, V. N. Strocov, and P. R. Willmott, D. Fontaine, Ph. Ghosez, A. Filippetti, P. Delugas, and V. Fiorentini, Phys. Rev. B 89, 121412 (2014).

[25] K. V. Shanavas, Z. S. Popović, and S. Satpathy, Phys. Rev. B, 90, 165108 (2014).

[26] N. C. Plumb, and M. Salluzzo, E. Razzoli, M. Månsson, M. Falub, J. Krempasky, C. E. Matt, J. Chang, M. Schulte, J. Braun, H. Ebert, J. Minár, B. Delley, K.-J. Zhou, T. Schmitt, M. Shi, J. Mesot, L. Patthey, and M. Radović, Phys. Rev. Lett. 113, 086801 (2014).

[27] A. Fête, S. Gariglio, C. Berthod, D. Li, D. Stornaiuolo, M. Gabay, and J.-M. Triscone, New J. Phys. 16, 112002 (2014).

[28] See Supplemental Material at http://link.aps.org/ supplemental/10.1103/PhysRevLett.115.016803 for additional experimental data, details on model parameters, point-like scatterers, effect of out-of-plane magnetic fields, and spin-orbit corrections to the Boltzmann transport.

[29] M. Salluzzo, J. C. Cezar, N. B. Brookes, V. Bisogni, G. M. De Luca, C. Richter, S. Thiel, J. Mannhart, M. Huijben, A. Brinkman, G. Rijnders, and G. Ghiringhelli, Phys. Rev. Lett. 102, 166804 (2009).

[30] J. M. Ziman, Principles of the Theory of Solids (Cambridge University Press, Cambridge, England, 1972).

[31] J. M. Ziman, Phys. Rev. 121, 1320 (1961).

[32] D. I. Pikulin, C.-Y. Hou, and C. W. J. Beenakker, Phys. Rev. B 84, 035133 (2011).

[33] S. Thiel, C. W. Schneider, L. F. Kourkoutis, D. A. Muller, N. Reyren, A. D. Caviglia, S. Gariglio, J.-M. Triscone, and J. Mannhart, Phys. Rev. Lett. 102, 046809 (2009).

[34] In the presence of strong spin-orbit interactions there can be additional corrections to Eqs. (8) and (9) $[35,36]$. We do not consider these here since we have found that they vanish for in-plane fields [28].

[35] N. A. Sinitsyn, Q. Niu, and A. H. MacDonald, Phys. Rev. B 73, 075318 (2006).

[36] N. Nagaosa, J. Sinova, S. Onoda, A. H. MacDonald, and N. P. Ong, Rev. Mod. Phys. 82, 1539 (2010).

[37] M. H. Fischer, S. Raghu, and E.-A. Kim, New J. Phys. 15, 023022 (2013).

[38] S. Thiel, G. Hammerl, A. Schmehl, C. W. Schneider, and J. Mannhart, Science 313, 1942 (2006). 